that the lesion may be the result of a hitherto unknown type of hypersensitivity response.

Our results do not show that the adverse effects of practolol are due to a hypersensitivity reaction, but two pieces of evidence might tentatively support such a conclusion. The first is the low antibody concentrations found in the sera of patients with sclerosing peritonitis. This might have been due to a reduction in antigenic stimuli, since the patients had been taken off the drug some time before the clinical signs of intestinal obstruction appeared. Equally, however, the results could be explained by postulating that the antigenic metabolite is incorporated into connective tissue and the mass of antigenic determinants presented by the peritoneum is sufficient to fix most of the circulating antibody. The second piece of evidence is perhaps more pertinent and concerns the challenge study on one of the patients (case 4). This patient had high concentrations of circulating antibody before challenge with practolol, and when the drug was given, clinical signs of the tissue damage were produced. Other patients in the study might have shown an adverse response if the drug had been given for a longer period.
Continued analysis of serum will probably provide only circumstantial evidence of a practolol-induced hypersensitivity reaction. An animal model is needed to study the tissue damage produced by the drug, and we are currently engaged in studies $\underline{\underline{T}}$ of this kind.

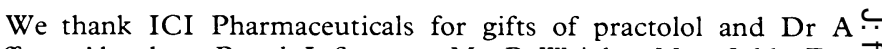
Jeffers, Aberdeen Royal Infirmary, Mr P Wright, Moorfields Eye Hospital, Dr F J Zacharias, Clatterbridge Hospital, and Dr S Pugh, Northwick Park Hospital, for providing serum samples.

\section{References}

${ }^{1}$ Amos, H F, Lake, B G, and Atkinson, H A C, Clinical Allergy, 1977, 7, 243.

2 Farr, R S, Fournal of Infectious Diseases, 1958, 103, 239.

Coombs, R R A, and Gell, P H G, in Clinical Aspects of Immunology, ed $\vec{\omega}$ P H G Gell and R R A Coombs. Oxford, Blackwell, 1963.

\title{
Diabetic cardiomyopathy? An echocardiographic study of young diabetics
}

\author{
J E SANDERSON, D J BROWN, A RIVELLESE, E KOHNER
}

British Medical fournal, 1978, 1, 404-407

\section{Summary and conclusions}

An echocardiographic study was carried out on 23 young diabetics, 19 of whom had retinopathy. Their diastolic function was analysed by comparing the timing and pattern of mitral valve opening with the pattern of left ventricular wall movement. Only six patients had all their values within the normal range. Fourteen patients had abnormalities similar to those seen in patients with cardiomyopathy; the close time relation between mitral valve movement and wall movement was lost and mitral valve opening delayed in eight patients. Three other patients had considerable outward wall movement before mitral valve opening, which is characteristic of ischaemic heart disease.

Although these studies provide no definite evidence of a cause, the abnormalities found may reflect a subclinical diabetic cardiomyopathy due to small-vessel disease.

\section{Introduction}

Accelerated coronary artery disease is a well-known feature of diabetes mellitus and it accounts for much of the morbidity and mortality of this condition. Several reports, ${ }^{1-3}$ however, have noted that congestive heart failure and cardiomegaly occur in

\footnotetext{
Hammersmith Hospital, London W12 0HS

J E SANDERSON, MA, MRCP, research fellow, department of clinical cardiology

A RIVELLESE, MD, research fellow, department of endocrinology

E KOHNER, MD, FRCP, senior lecturer, department of endocrinology

Cardiac Department, Brompton Hospital, London SW3

D J BROWN, BSC, MA, computer systems designer
}

diabetics with normal coronary arteries. At necropsy ${ }^{23}$ and in myocardial biopsy specimens ${ }^{1}$ small-vessel changes and diffuse $\overline{0}$

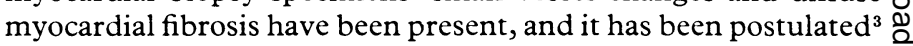
that a diabetic microangiopathy may cause this heart-muscle $\mathbb{\mathscr { Q }}$ disease or cardiomyopathy. ${ }^{2}$

Echocardiographic studies in healthy people have shown that diastole is a co-ordinated and precisely timed process. Relaxation $\vec{\partial}$ of the left ventricle and the consequent filling is reflected in the outward wall movement seen echocardiographically, ${ }^{4}$ and this movement is closely related to movement of the mitral valve, both events showing an identical pattern of movement. ${ }^{5}$. Disturbance of the close relation between left ventricular wall $\frac{0}{3}$ movement and mitral valve movement seems to be a sensitive index of a myocardial abnormality, and abnormalities have been $\frac{0}{3}$ shown in both ischaemic heart disease ${ }^{5}$ and hypertrophic cardiomyopathy. ${ }^{\circ}$; We therefore decided to perform echo- $\frac{0}{7}$ cardiographic studies to assess the myocardial function of young $\mathbb{\sim}$ diabetics. None had any clinical evidence of ischaemic heart $D$ disease, though most had retinopathy and therefore evidence of small-vessel disease. We also studied the beat-to-beat variation $N$ in heart rate as an indication of autonomic neuropathy affecting N the heart. ${ }^{8}$

\section{Patients and methods}

Echocardiographic studies were performed on 32 diabetics aged under 40 , but the records of only 23 were of sufficiently high standard $\stackrel{\mathbb{D}}{D}$ for further analysis. The mean age of these 23 patients (12 women) was 31 years (15-39 years). They had had diabetes for a mean of 17 years (2-28 years). All patients had normal blood pressure, were in sinus rhythm, and had no clinical evidence of heart disease. $ᄋ$ Sixteen patients had proliferative retinopathy, which was florid in one; three patients had background retinopathy and four had normal eyes. In 20 of these patients the beat-to-beat variation was also studied. All patients had a normal chest radiograph and electrocardiogram. Fifteen healthy subjects of the same age and sex distribution were also studied echocardiographically, and 18 healthy subjects had their beatto-beat variation measured. 

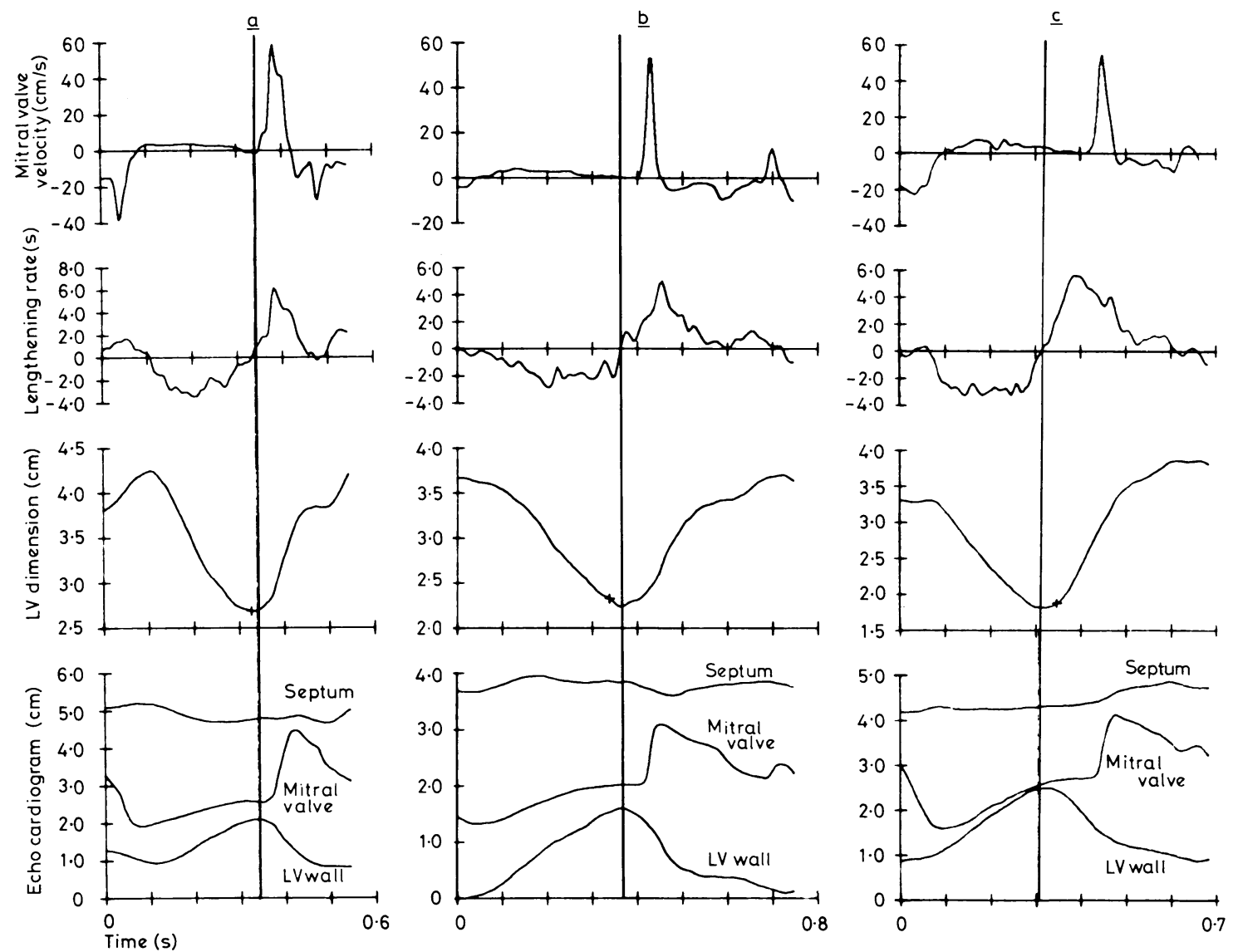

Plots of digitised echocardiograms showing change in left ventricular (LV) dimension (D), rate of change of dimension (dD/dt/D), and anterior mitral valve velocity. (a) Patient from group 1 with normal pattern. (b) Patient from group 3 with delay in mitral valve opening and disturbed relationship between LV filling and mitral valve movement. (c) Patient from group 5 with considerable outward wall movement before mitral valve opening.

Echocardiographic methods-Measurements were made with an EKOLINE 20 ultrasonoscope (frequency $2.25 \mathrm{MHz}$, repetition frequency $1000 / \mathrm{s})$. The output was displayed on a Cambridge multi hannel strip chart recorder at a paper speed of $100 \mathrm{~mm} / \mathrm{s}$, with a simultaneous electrocardiogram and phonocardiogram. The subjects were studied in the supine or left lateral position with the transducer at the left sternal border in the 3rd, 4th, or 5th interspace. The transducer was angled down from the aortic root through the mitral ring until echoes were obtained simultaneously from the septum, posterior wall, and the anterior leaflet of the mitral valve. Measurements were made only on those recordings showing clear continuous endocardial echoes from the septum and posterior wall.

Digitising methods-Echocardiograms were digitised as described. ${ }^{*}$ Echoes of the left ventricular edge of the septum, posterior left ventricular wall, anterior leaflet, or mitral valve were traced on a digitising table interfaced to a Prime computer. Strings of co-ordinates were generated for about 100 points from each of these echoes together with calibration signals representing 0.5 second, $1 \mathrm{~cm}$, and the R-R interval of the beat being studied. A timing mark representing the aortic component of the 2 nd heart sound $\left(\mathrm{A}_{2}\right)$ on the phonocardiogram was also inserted.

Analysis of data-The echocardiograms were analysed as described. ${ }^{5}$ From the stored data plots were made of $(a)$ the positions of the septum, left ventricular wall, and anterior mitral leaflet; $(b)$ instantaneous leaflet velocity; (c) left ventricular dimension (D); and $(d)$ the normalised rate of change of dimensions $(\mathrm{dD} / \mathrm{dt} / \mathrm{D})$ or lengthening rate (see figure). The relation of wall movement to mitral valve movement was assessed by measuring the intervals between the time of $(a)$ the onset of outward wall movement and that of mitral leaflet opening movement, $(b)$ the peak rate of change of dimension and peak opening velocity of the leaflet, and $(c)$ a discontinuity in the rate of wall movement previously shown to coincide with the end of rapid filling and a corresponding discontinuity in the leaflet velocity tracing during mid-diastolic closure. The end-diastolic dimension and peak lengthening rate were measured directly from the plots. If any outward movement occurred before the onset of mitral valve opening it was expressed as a percentage of the total diastolic dimension change during diastole.

Beat-to-beat variation-An ECG recording with a rhythm strip was carried out after the patients had been resting for 15 minutes in a quiet room. The recording was done for half a minute during normal breathing and for half a minute during deep breathing. The beat-to-beat variation was calculated as the difference between the maximum and minimum heart rates during deep breathing. ${ }^{8}$

\section{Results}

The normal subjects had values similar to those previously reported, ${ }^{45}$ and their mean values $\pm 2 \mathrm{SD}$ were taken as the normal range for each of the indices. On the basis of their electrocardiographic results the diabetic patients were divided into five groups (see table).

Echocardiographic data on five groups of diabetics and normal controls

\begin{tabular}{|c|c|c|c|c|c|}
\hline Group & $\begin{array}{c}\text { No of } \\
\text { patients }\end{array}$ & $\begin{array}{c}\text { Minimum } \\
\text { dimension } \\
\text { to onset of } \\
\text { mitral valve } \\
\text { movement } \\
\text { (ms) }\end{array}$ & $\begin{array}{l}\text { Peak } \\
\text { lengthening } \\
\text { rate to peak } \\
\text { opening } \\
\text { velocity of } \\
\text { mitral valve } \\
\text { (ms) }\end{array}$ & $\begin{array}{c}\text { Discontinuity } \\
\text { at end of } \\
\text { rapid filling } \\
\text { (dimension } \\
\text { trace) to } \\
\text { discontinuity } \\
\text { in anterior } \\
\text { mitral valve } \\
\text { tracing } \\
\text { (ms) }\end{array}$ & $\begin{array}{c}\text { Oo } \\
\text { Outward } \\
\text { wall } \\
\text { movement }\end{array}$ \\
\hline $\begin{array}{l}1 \\
2 \\
3 \\
4 \\
5\end{array}$ & $\begin{array}{l}6 \\
3 \\
5 \\
6 \\
3\end{array}$ & $\begin{array}{rl}7 \cdot 5 & =6 \\
41 \cdot 6 & =11 \\
31 & =7 \\
5 \cdot 8 & 6 \cdot 6 \\
92 & =20\end{array}$ & $\begin{array}{l}8 \cdot 3 \pm 11 \\
-15=5 \\
-46 \pm 29 \\
-31=20 \\
-10 \pm 53^{*}\end{array}$ & $\begin{aligned} &-7 \cdot 5=7 \\
&-5=13 \\
&-87 \pm 35 \\
&-85 \pm 44 \\
&-23 \pm 75\end{aligned}$ & $\begin{array}{c}0 \\
0 \\
0 \\
0 \\
21 \pm 10\end{array}$ \\
\hline Total & 23 & $27=30$ & $-29 \pm 22$ & $-51 \pm 47$ & $3 \pm 8$ \\
\hline $\begin{array}{l}\text { Normal } \\
\text { subjects }\end{array}$ & 15 & $3=6$ & $-6 \pm 8$ & $-7 \pm 10$ & $1 \cdot 4 \pm 2 \cdot 4$ \\
\hline
\end{tabular}

${ }^{*}$ Individual values were: $-30 \mathrm{~ms},+50 \mathrm{~ms}$, and $-50 \mathrm{~ms}$. 
Group 1 -The results in six patients were all normal. In early diastole there was a close relation between left ventricular wall and mitral valve movement, as in the normal subjects (see figure, $a$ ).

Group 2-In three patients the onset of mitral valve opening was significantly delayed with reference to minimum dimension, but there was no significant outward wall movement during this delay. After the mitral valve had started to open left ventricular wall movement and mitral valve movement showed similar patterns with a close time relationship.

Group 3-Five patients showed more severe abnormalities. The onset of mitral valve opening was significantly delayed and the peak lengthening rate occurred significantly later than the peak opening velocity of the mitral valve. In addition, left ventricular filling continued during the period of mid-diastolic closure of the mitral valve after the discontinuity in the leaflet tracing (see figure, $b$ ).

Group 4-Although the onset of mitral valve movement in these six patients was normal in relation to the onset of outward wall movement, rapid filling appeared to continue for longer. The peak lengthening rate did not, however, fall significantly in this group.

Group 5-Three patients showed a distinctive type of abnormality, which was similar to that described in patients with ischaemic heart disease and left ventricular wall dyskinesia." The characteristic pattern in left ventricular wall dyskinesia is of delayed mitral valve opening, but there is also a significant outward wall movement, which may account for $31 \%$ of the total diastolic excursion. All three patients in this group had significant outward wall movement while mitral valve opening was delayed. Again, the peak and discontinuity of the mitral valve velocity tracing were also significantly disturbed with respect to the corresponding events in wall movement. The figure $(c)$ shows that in one patient the degree of outward wall movement was consistent with a left ventricular segmental contraction abnormality. ${ }^{5}$ Indeed, this patient produced a result positive for ischaemia during a treadmill exercise test. The mean values of the whole group of patients were significantly abnormal $(\mathbf{P}<0.01)$ for the following indices: delay in onset of mitral valve movement, time between peak lengthening rate and peak mitral valve opening velocity, and time from the end of rapid filling on the dimension trace to the discontinuity on the mitral valve trace.

The end-diastolic dimension for all patients $(4.0 \pm 0.5 \mathrm{~cm})$ was normal $(4 \cdot 0 \pm 0.4 \mathrm{~cm})$, as was the peak lengthening rate $(4 \cdot 3 \pm 1 \cdot 1 \mathrm{~cm} / \mathrm{s}$; normal $4 \cdot 1 \pm 1 \cdot 2 \mathrm{~cm} / \mathrm{s})$. All patients had normal mitral valves.

Beat-to-beat variation-There was no significant difference in resting heart rate between normal subjects $(73 \pm 13$ beats $/ \mathrm{min})$ and diabetics $(79 \pm 14$ beats $/ \mathrm{min})$. There was, however, a significant difference in beat-to-beat variation. In the normal subjects the mean was $30 \pm 10$, while in diabetics it was $14 \pm 9(P<0.05)$. No normal subject had a variation below 12, while only eight of the 21 diabetic patients had such a small variation. There was no correlation between beat-to-beat variations and the different groupings in the echocardiographic study. Thus two patients in group 1, two patients in group 2, one in group 3, one in group 4, and two in group 5 had a beat-to-beat variation of 10 or less.

Clinical features-There was no association between the degree of retinopathy, the length of history or quality of previous diabetic control, and the type of diastolic abnormality.

\section{Discussion}

Early diastole is a part of the cardiac cycle that has received only scanty attention. But recent echocardiographic ${ }^{5}{ }^{6}$ and angiographic studies ${ }^{7} 910$ have emphasised the importance of this period on overall left ventricular function and shown that relaxation and filling of the ventricle is a precisely timed and co-ordinated process. Echocardiography has two advantages in the study of myocardial function: firstly, it is non-invasive and, secondly, the endocardium can be recognised unequivocally throughout diastole and the pattern of mitral valve cusp movement can be observed and compared with it. The versatility of echocardiography can be increased by using a simple digitising technique and computing the instantaneous mitral valve velocity and the left ventricular dimension and its rate of change, so that the relation between the mitral valve and wall movement can be determined more precisely.

This technique has shown that at the start of diastole a coordinated sequence of events occurs in normal people. After systole the left ventricular dimension reaches a minimum value and this point coincides with the onset of anterior movement of the mitral valve (see figure, $a$ ). The left ventricular dimension then increases rapidly during a period of acceleration lasting about 0.16 second. The rate of increase of dimension then drops abruptly so that by 0.22 second it has declined to $20^{\circ}{ }_{0}$ of its peak value, and there is a clear-cut discontinuity on the first derivative of the dimension trace, which represents the end of rapid filling and the onset of diastasis. ${ }^{11}$ Anterior mitral valve cusp movement is co-ordinated with that of the wall throughout this period. Not only the start but also the peak rate of anterior movement of the cusp coincides with that of the dimension, and both show a discontinuity at the end of rapid filling. During diastasis the mitral valve shows a characteristic mid-diastolic closure movement and reaches a peak closing velocity of 250 $\mathrm{mm} / \mathrm{s}$.

In all but six of our patients with diabetes and retinopathy the close time relations between wall movement and mitral valve movement were disturbed to some degree. In group 2 patients there was a delay in the onset of mitral valve opening, but once this had occurred the pattern of wall movement closely followed that of the mitral valve. A delay in mitral valve opening alone has been found in patients with ischaemic heart disease ${ }^{1: 2}$ and in those with hypertrophic cardiomyopathy ${ }^{6}$ In contrast, the onset of mitral valve movement in patients in group 4 occurred promptly at minimum dimension but the filling pattern was abnormal, with outward wall movement continuing during middiastolic closure of the mitral valve. It is difficult to be certain of the significance of the abnormalities in these groups as they are only minor. But patients in group 3 seemed to have a more severe disorganisation, with a combined delay in mitral valve opening and a disturbed relationship between the mitral valve and left ventricular wall (figure, $b$ ). This is a pattern of abnormality that has been found in hypertrophic cardiomyopathy, ${ }^{6}$ a condition in which diastole is abnormal. The three patients in group 5 showed a pattern associated with ischaemic heart disease, with considerable outward wall movement taking place while mitral valve opening was delayed (figure, $c$ ). All three patients in this group underwent a treadmill exercise test and the patient with the largest degree of outward wall movement had a result positive for ischaemia.

Our present observations cannot provide an explanation for these abnormalities, but they do seem to suggest that some diabetics with retinopathy have abnormalities of diastolic function that may reflect subclinical heart disease. It is unlikely that all the abnormalities seen were due to premature coronary artery disease in view of the youth of our patients, their lack of symptoms, and their different patterns of abnormal diastolic function. The similarity of the abnormalities of the patients in group 3 to those found in patients with hypertrophic cardiomyopathy suggests a possible cause of the abnormalities. The possibility of a diabetic cardiomyopathy has been suggested ${ }^{1-3}$ to explain the development of heart failure in diabetics with normal coronary arteries who have evidence of small-vessel disease in the myocardium. Most of our patients had evidence of severe small-vessel disease in their retina, and, although there was no clear relation between the severity of abnormalities and the degree of retinopathy, these patients may also have had smallvessel disease in their myocardium. Clearly a spectrum of abnormalities is possible and our results may reflect an interaction between a diabetic small-vessel cardiomyopathy and the premature onset of coronary artery disease.

JES was supported by the Wellcome Trust, and AR by the Hoechst Trust. The computer equipment used in the study was provided by the DHSS as part of their research programme. We thank Professor J F Goodwin for his help and encouragement.

\section{References}

${ }^{1}$ Pearce, M B, Bulloch, R T, and Kizziar, J C, Circulation, 1973, 38, suppl No $4, \mathrm{p} 6$. 
${ }^{2}$ Hamby, R I, Zungraich, S, and Sherman, L, fournal of the American Medical Association, 1974, 229, 1744.

${ }^{3}$ Rubler, S, et al, American fournal of Cardiology, 1972, 30, 595.

4 Gibson, D G, and Brown, D J, British Heart Fournal, 1973, 35, 1141.

5 Upton, M T, Gibson, D J, and Brown, D J, British Heart fournal, 1976, 38, 1001 .

6 Sanderson, J E, et al, British Heart fournal, 1977, 39, 661.

7 Sanderson, J E, et al, British Heart fournal. In press.

${ }^{8}$ Wheeler, T, and Watkins, P J, British Medical fournal, 1973, 4, 584.
${ }^{9}$ Gibson, D G, Prewitt, T A, and Brown, D J, British Heart Fournal, 1976, 38, 1010.

${ }^{10}$ Ruttley, M S, et al, Circulation, 1974, 50, 306.

11 Straub, H, Fournal of Physiology, 1910, 40, 378.

12 Rubinstein, J J, Pohost, G M, and Foster, J R, Clinical Research, 1973, 21, 446 .

(Accepted 28 November 1977)

\section{SIDE EFFECTS OF DRUGS}

\section{Levamisole-induced vasculitis due to circulating immune complexes}

The immunostimulant drug levamisole is being assessed in the treatment of many conditions, including rheumatoid arthritis. ${ }^{1}$ Reports of side effects are accumulating, especially in patients with immunological disorders. In one group of 69 patients with cancer receiving immunotherapy with levamisole, three developed non-

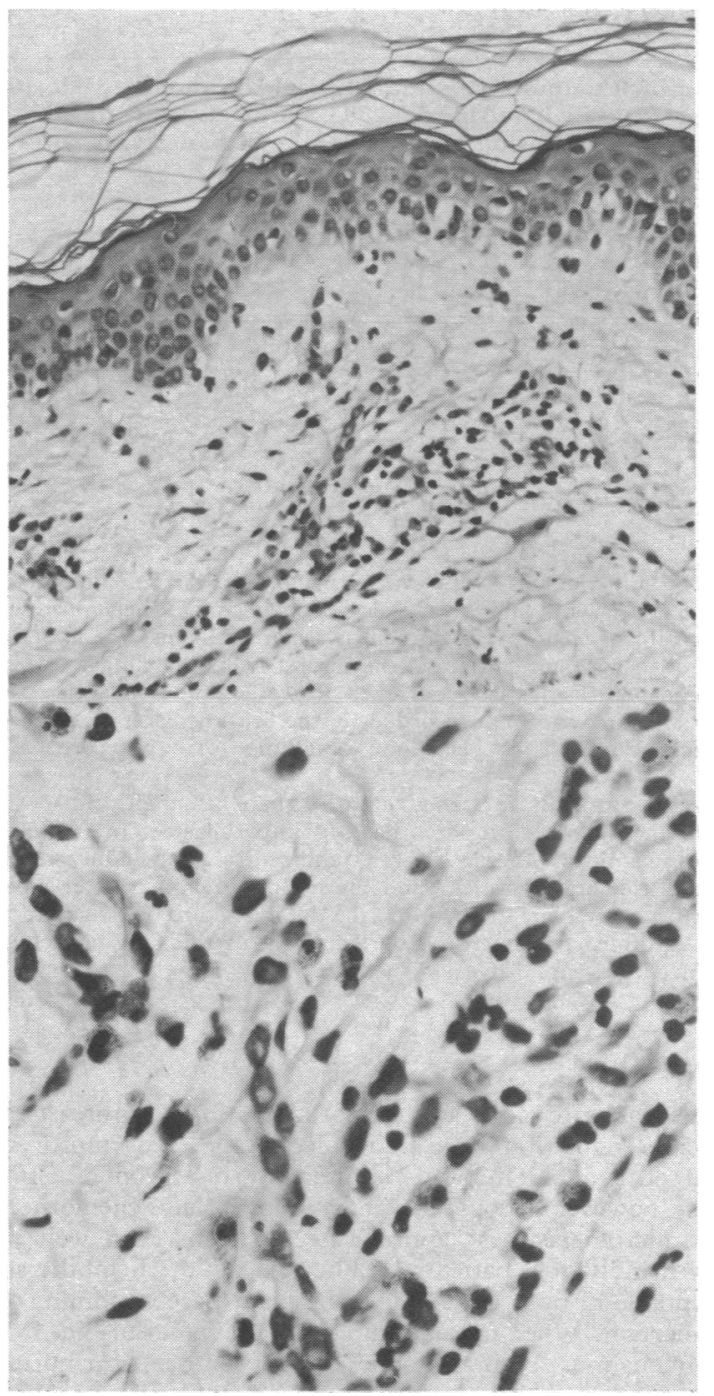

Biopsy specimen of forearm skin. Above: Low-power view showing leucocytoclastic vasculitis with inflammatory cells and nuclear debris around small cutaneous vessels. (Haematoxylin and eosin. $\times 500$.) Below: Highpower view showing prominent eosinophils. ( $H$ and $\mathrm{E} . \times 1250$.) specific rashes. ${ }^{2}$ We describe a patient who developed a cutaneous leucocytoclastic vasculitis while receiving levamisole for rheumatoid arthritis, a hitherto unrecognised complication.

\section{Case report}

A 65-year-old woman with seropositive rheumatoid arthritis of 10 years' duration began treatment with $150 \mathrm{mg}$ levamisole daily. She was also taking enteric-coated aspirin (Nu-seals) and occasional Distalgesic tablets (dextropropoxyphene hydrochloride and paracetamol). Two months later, while continuing on this dose of levamisole, she developed a widespread rash with a livedo pattern, chiefly on the arms and legs; she was otherwise well and thought that the arthritis had improved. Biopsy of a typical lesion on the forearm showed a leucocytoclastic vasculitis with predominant eosinophils (see figure).

Immunofluorescence showed IgM in the basement membrane and C3 around the vessels. The haemoglobin was $11.8 \mathrm{~g} / \mathrm{dl}$; white cell count was $7 \cdot 0 \times 10^{9} / 1\left(7000 / \mathrm{mm}^{3}\right)$; platelets were plentiful; and ESR was $27 \mathrm{~mm}$ in the first hour. Serum complement concentrations $\left(\mathrm{CH}_{50}, \mathrm{C} 3\right.$, and $\left.\mathrm{C} 4\right)$ were normal. Circulating immune complexes were not detected by measuring anticomplement activity or by the platelet aggregation test. Nevertheless, a histamine skin-weal test ${ }^{3}$ reproduced the leucocytoclastic vasculitis in clinically unaffected site. Biopsy of control, uninjected skin showed increased numbers of eosinophils around the vessels but no vasculitis. Immunofluorescence of the histamine-injected site showed IgM and complement, as in the clinically affected skin, but these were absent in the control specimen. The rash rapidly disappeared when levamisole was withdrawn. One month later the histamine skin test was repeated. The uninjected skin was normal, but the injected site showed infiltration of round cells, with immunoglobulins and complement around the vessels.

\section{Comment}

Levamisole was apparently responsible for the vasculitic rash since the condition rapidly disappeared on withdrawal of the drug, though the other treatment was unchanged. Immunological mechanisms were clearly important. Although serological tests for circulating immune complexes gave negative results, the histamine skin test produced a reaction. This was possibly because histamine allowed sufficient immune complexes to accumulate in one site to trigger complementmediated damage and thus reproduce the vasculitis.

Levamisole-induced agranulocytosis has also been shown to be due to immunological mechanisms by the finding of leucocytotoxic agglutinins. ${ }^{4}$ Arthritis induced by levamisole has been reported in Crohn's disease ${ }^{5}$ with negative serological tests for immune complexes. As in Crohn's disease, our patient developed a well-recognised complication of the primary disease while on levamisole. Clearly doctors cannot dismiss the appearance of a complication as being due to the disease alone in patients receiving levamisole unless it persists after withdrawal of the drug. Our patient's abnormal response to the histamine test one month after stopping treatment suggests that immune complexes were persisting, but not in sufficient quantity to cause a clinical vasculitis. This supports the possibility that levamisole produces complications by unmasking pre-existing but latent immunological abnormalities rather than initiating disease. Further reports of side effects of levamisole will shed more light on both the mode of action of the drug and the diseases it is used to treat.

We thank Dr St John Dixon for permission to report this case and Dr Pat Burton for the histological report.

1 Huskisson, E C, et al, Lancet, 1976, 1, 393.

2 Parkinson, D R, et al, Lancet, 1977, 2, 1129. 